\title{
Emotion recognition using eigenvalues and Levenberg-Marquardt algorithm-based classifier
}

\author{
VILAS H GAIDHANE ${ }^{1, *}$, YOGESH V HOTE ${ }^{2}$ and VIJANDER SINGH ${ }^{3}$ \\ ${ }^{1}$ Department of Electrical and Electronics Engineering, BITS Pilani, Dubai Campus, Dubai International \\ Academic City, Dubai 34505, UAE \\ ${ }^{2}$ Department of Electrical Engineering, Indian Institute of Technology Roorkee, Roorkee 247667, India \\ ${ }^{3}$ Department of Instrumentation and Control Engineering, Netaji Subhas Institute of Technology, University of \\ Delhi, New Delhi 110078, India \\ e-mail: vilasgd612@gmail.com
}

MS received 17 August 2013; revised 4 December 2014; accepted 17 February 2016

\begin{abstract}
In this paper, a simple and computationally efficient approach is proposed for person independent facial emotion recognition. The proposed approach is based on the significant features of an image, i.e., the collection of few largest eigenvalues (LE). Further, a Levenberg-Marquardt algorithm-based neural network (LMNN) is applied for multiclass emotions classification. This leads to a new facial emotion recognition approach (LE-LMNN) which is systematically examined on JAFFE and Cohn-Kanade databases. Experimental results illustrate that the LE-LMNN approach is effective and computationally efficient for facial emotion recognition. The robustness of the proposed approach is also tested on low-resolution facial emotion images. The performance of the proposed approach is found to be superior as compared to the various existing methods.
\end{abstract}

Keywords. Emotion recognition; eigenvalue; Levenberg-Marquardt algorithm; neural network; classification.

\section{Introduction}

Over the last 20 years, there has been a growing interest in the field of human computer interaction. In the case of human to human interaction, it is discovered that verbal cues provide $7 \%$, vocal cues, $38 \%$, and facial expressions $55 \%$ meaning of the message $[1,2]$. Thus, the facial expression provides more information than the thousand spoken words. The visual perception of human emotion is mainly focused on the facial expression of emotions. Deriving an effective facial expression representation from the original face image databases is an interesting and challenging problem.

Extracting the facial features from the face images is an important step for facial emotion recognition. There are two basic methods to extract the facial features, i.e., geometric features-based methods and appearance-based methods [3]. Geometric features contain information about the shape and location of the local features such as mouth, nose and eyes. These local feature statistics and locations are used for the classification. On the other hand, appearance-based methods extract the features of whole face region. They consider all the pixels of a face image and represent it by a vector space matrix. However, these vector space matrices are very large, which increases the computational complexity

*For correspondence in the system [4]. Although, geometric features-based methods provide better performance than the appearancebased method, these methods are more sensitive to noise and tracking errors.

Automatic facial expression recognition involves facial features representation and classification. Facial features representation is used to calculate a set of features from the original face images. In literature, optical flow analysis has been used to estimate the displacement of feature points [57]. Facial geometry-based analysis has been widely used in facial expression representation, where the shapes and location features are extracted to represent the face geometry $[8,9]$. However, the geometry-based method requires accurate and reliable feature detection which is a difficult task in many applications.

Another method to represent the facial expression is the appearance-based method, which uses spatial analysis. Principal component analysis [10], linear discriminant analysis [11, 12], independent component analysis [13] and wavelet analysis have been commonly used to extract the facial features. Due to the superior performance, Gabor wavelet method has been widely exploited in the facial expression analysis [14]. However, it requires large memory space and higher computational time. In recent years, local binary patterns (LBP) method has been introduced for appearance-based face analysis which requires comparable memory space and computational time [15]. 
The LBP operator was introduced by Ojala et al $[16,17]$. The LBP operator uses the neighborhoods of $3 \times 3,8 \times 8$ or $16 \times 16$ pixels size. In this, operator labels the pixels $p_{q}(q=0, \ldots, 7)$ of an image by thresholding each pixel with the value of center pixel $p_{c}$. An illustration of LBP operator is shown in figure 1.

The values in each pixel are thresholded with the center pixel value and a binary number is extracted.

$$
B\left(p_{q}-p_{c}\right)= \begin{cases}1 & \text { if } p_{q} \geq p_{c} \\ 0 & \text { otherwise }\end{cases}
$$

The LBP can be computed as follows:

$$
L B P=\sum_{q=0}^{7} B\left(p_{q}-p_{c}\right) 2^{q},
$$

where $2^{q}$ is a binomial factor for each $B\left(p_{q}-p_{c}\right)$. LBP has proven to be an effective method in appearance-based face representation and it has been applied to motion detection, visual inspection and scene analysis [18].

Face images consist of micro-patterns which can be described by LBP histograms and computed over the entire face without any indication of pattern locations. Shan et al [15] performed person independent facial emotion recognition using LBP features and different machine learning techniques such as template matching, support vector machines, linear discriminant analysis and linear programming. However, the LBP-based methods are complex as the thresholding and histogram of selected small subdivision is the part of initial process of LBP operator.

In the past, different techniques have been proposed to classify facial emotions, such as neural network [19, 20], support vector machines (SVM) [21], Bayesian network [22], and fuzzy rule-based classifiers [23, 24]. The Hidden Markov Model (HMM) is also proposed to model the temporal behavior of facial emotions from the image sequence [25]. However, HMMs cannot deal with the dependencies in observations. Therefore, a new approach for facial expression recognition based on the property of larger eigenvalues of an image and LM-based neural network is proposed.

In this paper, a new facial emotion recognition approach based on the larger eigenvalues and LM-based classifier is proposed. The larger eigenvalues of a diagonal matrix are less affected by noise and contrast whereas smaller eigen-

\begin{tabular}{|c|c|c|c|c|c|c|}
\hline 110 & 124 & 185 & \multirow[b]{2}{*}{ Thresholding } & 0 & 0 & 1 \\
\hline 195 & 170 & 165 & & 1 & & 0 \\
\hline 105 & 255 & 125 & & 0 & 1 & 0 \\
\hline
\end{tabular}
values are more sensitive to noise. Therefore, by using only

Figure 1. The basic LBP operator (Binary number $=00100101$, Decimal number $=37$ ). few larger eigenvalues, one can effectively represent the dynamics of face images [26]. The motivation of presented work is to propose a technique which requires less memory space and computational time as compared to Gabor filter and LBP methods.

The rest of the paper is structured as follows. A brief overview of existing LBP method is given in section 1. Section 2 describes the proposed approach for facial emotions recognition. Section 3 defines the details of JAFFE and CohnKanade database and the experimental results are explained in section 4. Finally, conclusions are drawn in section 5.

\section{Proposed approach for facial emotion recognition}

\subsection{Eigenvalues computation}

In this paper, the larger eigenvalues are considered for important features representation of face images. Therefore, before proceeding to the proposed approach, it is necessary to formulate eigenvalue-based problem which is used for facial emotion representation. The generalized Rayleigh quotient for optimization is given as Wee and Parmesran [26], Bie et al. [27],

$$
\Re(w)=\max _{w} \frac{w^{T} A w}{w^{T} B w},
$$

where $A, B \in \Re^{n \times n}$ are both symmetric matrices and $B$ is positive definite and hence invertible. Moreover, $w$ is the nonzero scaling vector and it does not change the value of the object (face image) matrix $A$. Thus, one can impose an additional scalar constraint on $w$ and optimize the object matrix $A$ in the form $B$ without any loss of information. The constraint is chosen to be unity matrix as $w^{T} B w=1$. One should note that the eigenvalues can be obtained for square, symmetric and definite matrix only and therefore, the face image matrix $A$ and its optimized matrix $B$ should be in such compatible forms.

The numerator of Eq. (3) produces a matrix whose eigenvalues are the diagonal elements which can be represented as

$$
\begin{aligned}
w^{T} A w & =w^{T} B w \Lambda, \\
& =I \Lambda=\Lambda .
\end{aligned}
$$

where $\Lambda=\operatorname{diag}\left(\lambda_{1}, \lambda_{2}, \ldots, \lambda_{n}\right)$ and $\lambda_{1} \geq \lambda_{2} \geq \cdots \geq \lambda_{n}$.

Here, most of the important dynamics of an image are represented along the direction indicated by the larger eigenvalues whereas the smaller eigenvalues are more sensitive to noise [26]. Thus, by using few larger eigenvalues, the dynamics of interest of an image with minimum effect of noise and resolution can be represented. This also reduces the dimension and computational complexity without extensive deterioration. Therefore, in this paper, an attempt has been made and only six largest eigenvalues are 
considered for statistical feature representation of face images.

\subsection{Emotion classification}

SVM has been extensively used as a classification method and successfully applied to facial emotions classification [6, 15, 21]. However, SVM makes only binary decisions and so, multiclass classification is accomplished by using oneto-all method. Therefore, it is necessary to suggest a new method for efficient multiclass classifications. In literature, the neural networks are widely used in the field of facial expression recognition for classification [19, 20, 28]. In these methods, numerical optimization techniques-based learning is generally suitable for classification task. However, the selection of the optimization techniques is totally depends on the nature of error function. The LevenbergMarquardt algorithm is a modification of Newton's method specially designed for minimizing the error function that is sum of squares of non-linear functions [29-32]. This is very well suited to neural network training, where the mean squared error is used as the performance index. The architecture of three-layer LM-based neural network is shown in figure 2.

The main aim of the Levenberg-Marquardt-based neural network is to learn the relations between a set of input vector $p_{q}$ and target output $t_{m}$ pairs; $\left[\left(p_{1}, t_{1}\right),\left(p_{1}, t_{2}\right)\right.$, $\left.\cdots,\left(p_{1}, t_{m}\right)\right],\left[\left(p_{2}, t_{1}\right),\left(p_{2}, t_{2}\right), \cdots,\left(p_{2}, t_{m}\right)\right], \cdots, \quad\left[\left(p_{q}, t_{1}\right)\right.$, $\left.\left(p_{q}, t_{2}\right), \cdots,\left(p_{q}, t_{m}\right)\right]$. The mean square error (MSE) is the performance measure of the neural network. Suppose, $F(x)$ is squared error function then

$$
F(x)=\sum_{i=1}^{N} e_{i}^{2}(x)=\sum_{i=1}^{m} \sum_{j=1}^{q}\left(t_{i}-p_{j}\right)^{T}\left(t_{i}-p_{j}\right),
$$

where $e(x)$ is the square error between the input vector $p_{q}$ and the target output $t_{m}$ of the neural network, and $N=1,2,3, \ldots, q \times m$. Here, $x$ is the vector of all the weights and threshold and $F(x)$ is to be minimized with respect to the parameter vector $x$.

The gradient $\nabla F(x)$ is given as

$$
\nabla F(x)=2 J^{\mathrm{T}}(x) e(x)
$$

where $J$ is the Jacobian matrix. Suppose, $x_{k}$ is the value for $k$ th iteration, then $x_{k+1}$ for $(k+1)^{\text {th }}$ iteration can be calculated as

$$
\begin{aligned}
& x_{k+1}=x_{k}-S_{k}^{-1} g_{k} \\
& x_{k+1}=x_{k}-\left[2 J^{T}\left(x_{k}\right) J\left(x_{k}\right)\right]^{-1} 2 J^{T}\left(x_{k}\right) e\left(x_{k}\right), \\
& x_{k+1}=x_{k}-\left[J^{T}\left(x_{k}\right) J\left(x_{k}\right)\right]^{-1} J^{T}\left(x_{k}\right) e\left(x_{k}\right) .
\end{aligned}
$$

where $S_{k}^{-1}=\left.\nabla^{2} F(x)\right|_{x=x_{k}}$ is called as Hessian matrix and $g_{k}=\left.\nabla F(x)\right|_{x=x_{k}}$ is the gradient. Eq. (7) is called as GaussNewton method. Further, the Hessian matrix $S_{k}^{-1}$ can be approximated as

$$
S_{k}^{-1}=\nabla^{2} F\left(x_{k}\right)+\mu_{k} I .
$$

Then the LM algorithm can be approximated as

$$
\Delta x_{k}=-\left[J^{T}\left(x_{k}\right) J\left(x_{k}\right)+\mu_{k} I\right]^{-1} J^{T}\left(x_{k}\right) e\left(x_{k}\right) .
$$

This algorithm has very important feature such that as $\mu_{k}$ increases, it approaches the steepest decent algorithm and as $\mu_{k}$ decreases to zero, the algorithm become Gauss-Newton. Generally, the LM algorithm begins with $\mu_{k}=0.01$. If a step does not reach to smaller value for $F\left(x_{k}\right)$, then the step is repeated by multiplying the $\mu_{k}$ by factor $\sigma \geq 1$. This approaches to the steepest descent but results into slow convergence. On the other hand, if a step produces a smaller value for $F\left(x_{k}\right)$, then the $\mu_{k}$ is divided by $\sigma \geq 1$ for the next step so that the algorithm becomes Gauss-Newton and provides the faster convergence. The MSE is proportional to the sum of squared error over the $\Phi$ targets in the training set. The error function $F\left(x_{k}\right)$ is highly nonlinear and therefore, Levenberg-Marquardt algorithm is best suited for

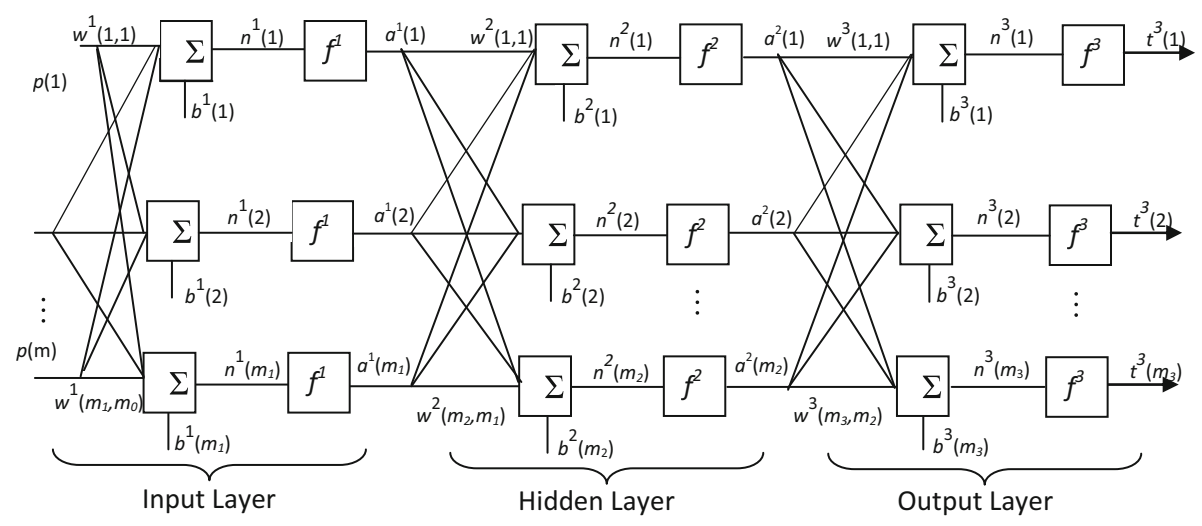

Figure 2. The basic architecture of LM-based neural network. 
nonlinear functions. Thus, the Levenberg-Marquardt algorithm can be a correct solution for above nonlinear error function optimization. Therefore, it is straightforward adapted for training. Thus, Eq. (5) evaluates the performance of the LM-based neural network classifier [33].

Using the above mathematical analysis, a facial emotion recognition approach based on the larger eigenvalues extracted from the image matrix and LM-based neural network is proposed. The face image has been normalized before proceeding to make face image contrast independent. The proposed approach is summarized in the following steps:

Step 1: Normalized the face image by its energy using the formula

$$
\tilde{r}(i, j)=\frac{r(i, j)}{\sqrt{\sum_{i=1}^{N} \sum_{j=1}^{M}[r(i, j)]^{2}}},
$$

where $r(i, j)$ is the $N \times M$ gray-scale test image.

Step 2: Compute the mean operator $\mu$ of the normalized test image $\tilde{r}(i, j)$ as

$$
\mu=\frac{1}{N \times M} \sum_{i=1}^{N} \sum_{j=1}^{M} \tilde{r}(i, j) .
$$

Step 3: Compute the covariance matrix $[C]$ as

$$
[C]=\frac{1}{N-1} R R^{\mathrm{T}},
$$

where $R=\tilde{r}-\mu$. This step converts the face image matrix into symmetric matrix.

Step 4: Suppose the eigenvalues of $[C]$ are represented as $\Lambda$, then they can be calculated using singular value decomposition (SVD) in descending order.

Step 5: Obtain the first $n$ diagonal eigenvalues of the above matrix, i.e., $\Lambda_{n}$ where, $n=6$.

Step 6: Calculate $\Lambda_{n}$ for all images available in image database using step 1 to step 5 and form the vector space $p_{q}$ for facial emotion classification.

Step 7: Repeat the step 1 to 6 for target images and form a vector space $t_{m}$.

Step 8: Classify and test the emotions using the Levenberg-Marquardt (LM)-based neural network.

The whole process of emotions recognition is summarised using the flow chart as shown in figure 3 .

The largest eigenvalues mainly represents the dominant features of the face image and are less sensitive to noise and blur effects. After detailed study and analysis on image eigenvalues, it is observed that the magnitude of first few (1 to 10) eigenvalues are significant and the magnitude of remaining eigenvalues is very small or zero. Therefore, in proposed approach first six largest eigenvalues has been used to represent the face image features. Since the larger

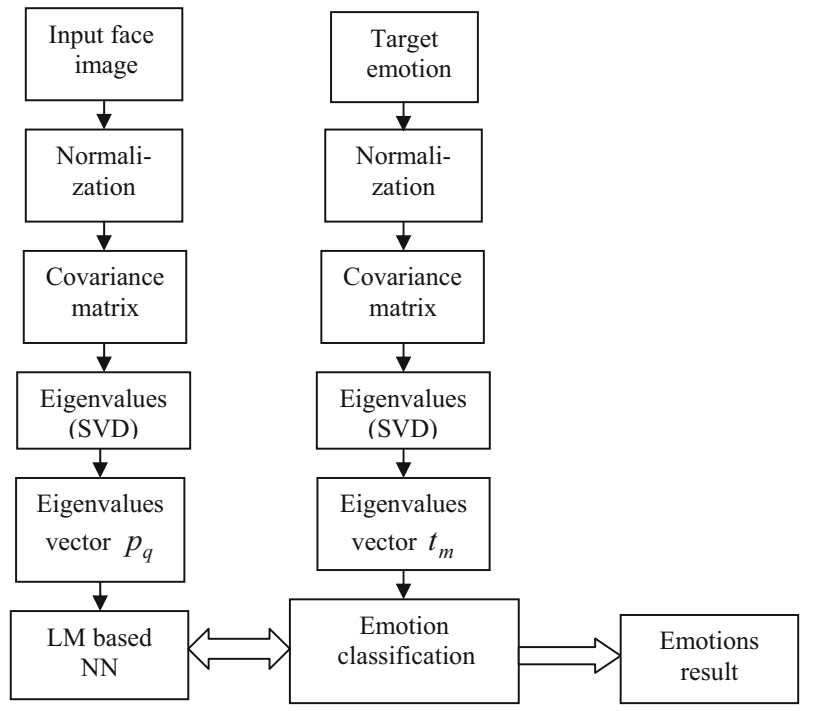

Figure 3. Block diagram for presented algorithm.

eigenvalues are independent of noise and resolution effect, the proposed approach is monotonic.

\section{Facial expression database}

Popular databases for facial emotions recognition are JAFFE database [34] and the Cohn-Kanade database [35]. The JAFFE database contains 213 images of seven facial expressions posed by 10 Japanese female models. Each image size is of $256 \times 256$ pixels. The Cohn-Kanade database is another most popular database used for facial expression recognition which includes 486 sequences from 97 posers. Each sequence of face images begins with a neutral expression and proceeds to peak expressions. All the images in Cohn-Kanade database are of the size $640 \times 490$ pixels.

Physiological studies indicate that the basic emotions have some universal facial expressions across all cultures and most of the recognition systems attempt to recognize a set of these prototypic emotional expressions such as Anger, Disgust, Fear, Joy, Sadness, Surprise and Neutral [23]. For experimentations, JAFFE database of 213 images of seven facial expressions posed by 10 Japanese female models and 320 image sequences from Cohn-Kanade database are considered. The sample face images from the JAFFE database and Cohn-Kanade database are shown in figure 4 and figure 5 , respectively.

\section{Experimental results and discussion}

The facial emotions recognition method based on larger eigenvalues and LM-based neural network classifier (LELMNN) is applied to the databases discussed above. The experimental results are described and analyzed in the following subsections. 

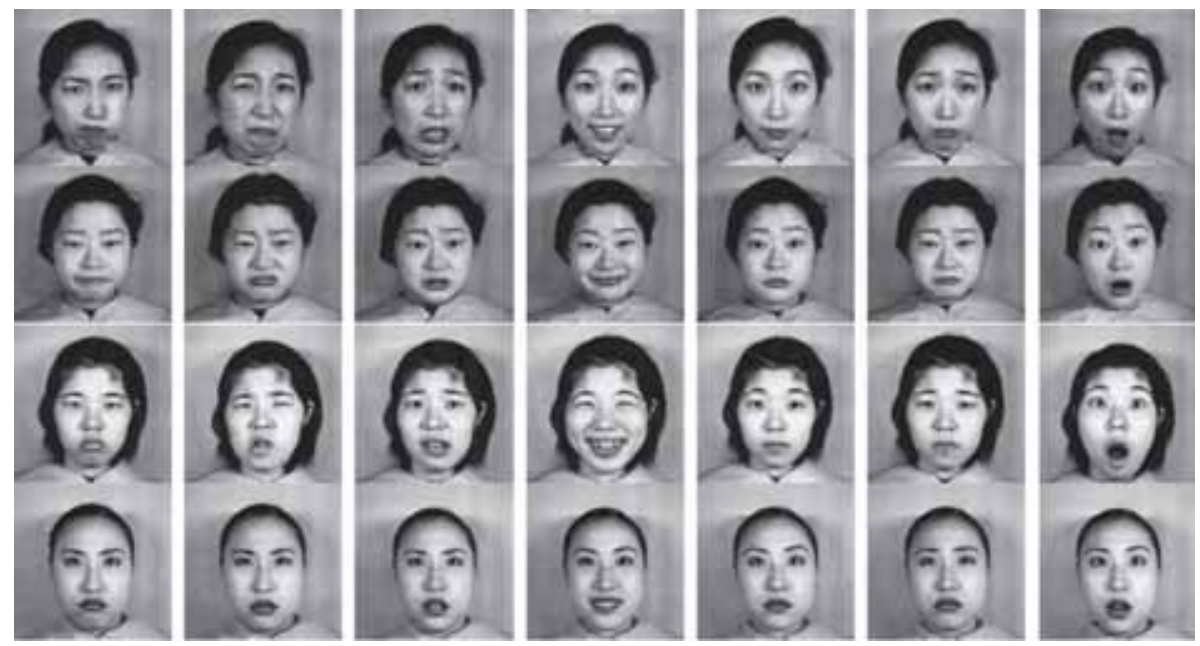

Figure 4. The sample face expression images from JAFFE database.
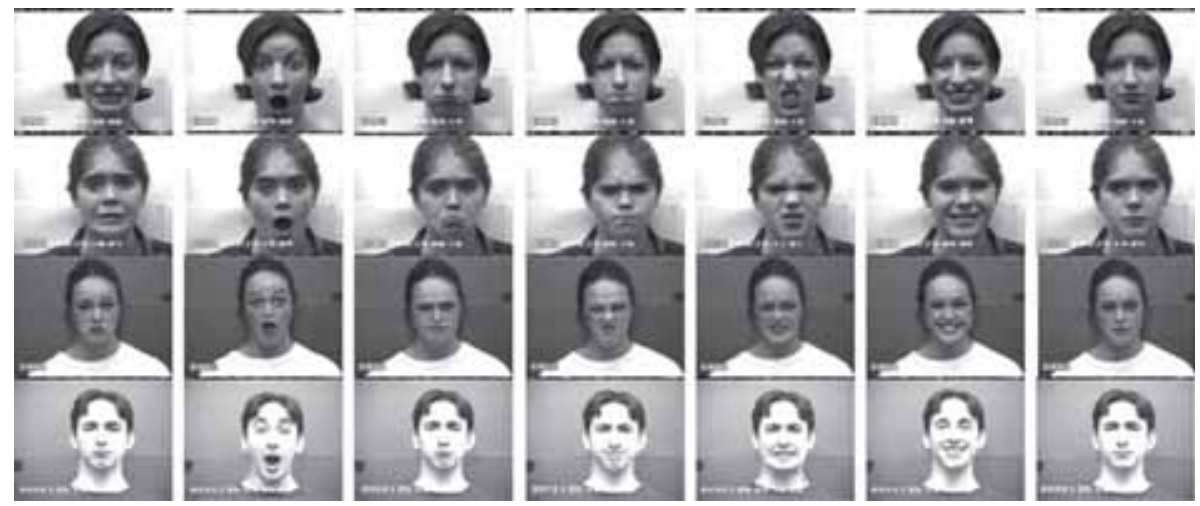

Figure 5. The sample face expression images from Cohn-Kanade database.

\subsection{Experimentation on the JAFFE database}

The LE-LMNN approach is applied on publicly available JAFFE database [34]. The JAFFE database consists of 213 images of Japanese models. This dataset is formed for seven-class expressions recognition (six emotional expressions and one neutral face).

In this experiment, all 213 images of the JAFFE dataset are used for seven-class expression recognition. The face images are normalized to fix the distance between two eyes and these images are cropped to the size of $128 \times 128$ pixels. To evaluate the expression recognition, a 10 -fold cross-validation testing scheme has been adapted for the various experimentations. In these experiments, the entire dataset is partitioned randomly into 10 groups with equal number of images. Further, nine groups are used as a training database to train the neural network classifier where as one group is used as a test dataset. One should note that the 10 -fold cross-validation scheme give the optimum performance only for larger datasets.
First of all, the appearance-based facial expression extraction is performed using the proposed eigenvaluebased approach. For each image, $\Lambda_{n}(n=6)$, six larger eigenvalues are obtained. Further, the Levenberg-Marquardt-based neural network with 10 neurons in the input layer, 10 neurons in each of the two hidden layers and 7 neurons in the output layer is used. Initially, $\mu_{k}$ is set to 0.001 . If a step does not reach to smaller value for error function then the step is repeated by multiplying the $\mu_{k}$ by the increase factor 10. This approaches to the steepest descent but results into slow convergence. On the other hand, if a step produces a smaller value for error function, then the $\mu_{k}$ is decreased by factor 0.1 for the next step so that the algorithm will move towards the Gaussian-Newton, which provides the faster convergence. The maximum value of $\mu_{k}$ is set to 10 with 0.1 minimum performance gradient.

The seven outputs of the neural network represent the seven different emotions. The training space consists of different sets of $\Lambda_{n}$. The testing of the seven-class emotions 


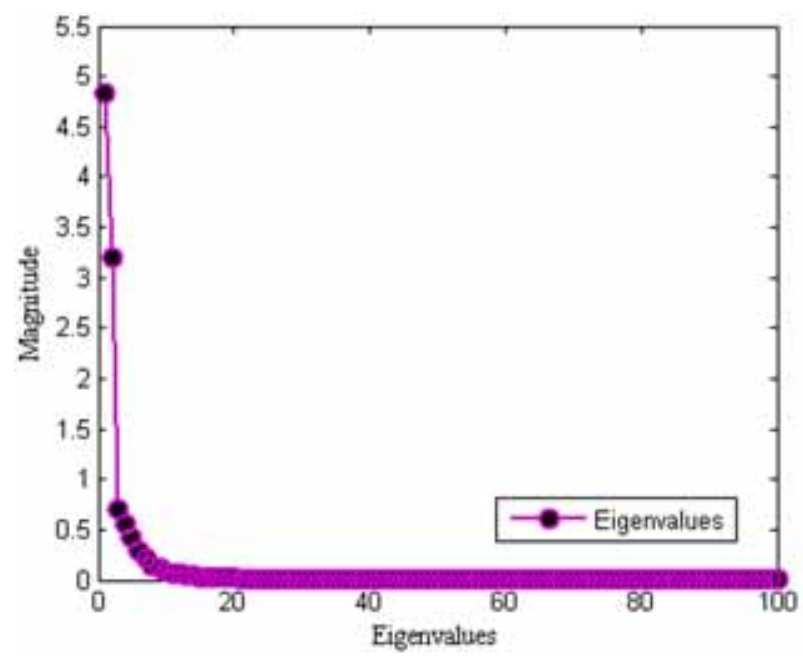

Figure 6. The plot of magnitude of eigenvalues in descending order.

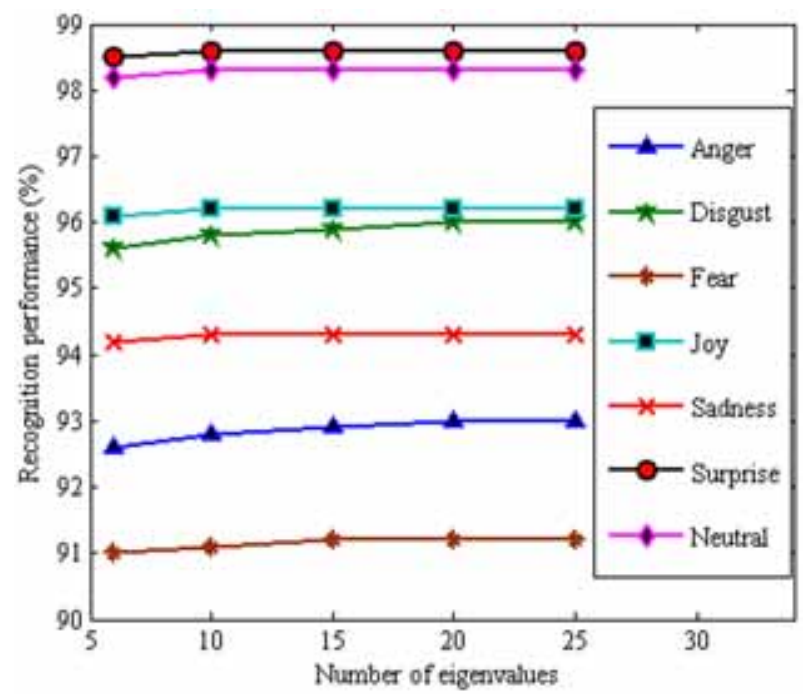

Figure 7. Variation of recognition performance of different emotions.

is carried out by calculating the closeness of desired $p_{q}$ values and $t_{m}$ vector space obtained from the training and testing datasets, respectively.
The variation of magitude of eigenvalues of a image covarience matrix $\boldsymbol{C}$ for a first individual of JAFFE database (figure 3) is shown in figure 6. It is observed from figure 6 that the convergence of magnitude of eigenvalues is faster and become zero after some larger eigenvalues. Therefore, most of the features of an image can be represented efficiently using few nonzero eigenvalues.

It is reported in literature that [4] the recognition accuracy of face images can be very with respect to the change in number eiegenvalues. Therefore, the recognition accuracy is obtained using the proposed approach for different number eigenvalues. The comparison of recognition performance of various emotions at the different number of eigenvalues is shown in figure 7.

From figure 7, it is observed that the recognition performance of proposed approach is less affected by the variation in number of eigenvalues. Moreover, recognition performance of Surprise and Neutral emotion is higher than the other emotions as they are more distingushabale from rest of the emotions.

In general, the emotions recognition performance is presented in the form of confusion matrix. A confusion matrix is a specific table layout that allows visualization of the performance of an algorithm, specifically supervised learning. Table 1 shows the confusion matrix of seven-class facial emotions recognition for JAFFE database. It is observed that Disgust, Joy, Neutral and Surprise emotions are recognized with high accuracy (94.4-96.5\%) while the recognition rates for Anger, Fear and Sadness are much lower (91.0-94.2\%).

Table 2 shows the comparison of recognition performance of the reported methods and the LE-LMNN approach for JAFFE database. It is observed from tables 1 and table 2 that the LE-LMNN approach provides significantly higher recognition performance as compared to the reported methods [36, 37]. As the proposed method suits well to the JAFFE database, it is also applied on CohnKanade database and discussed in the next subsection.

\subsection{Experimentation on the Cohn-Kanade database}

In this experiment, the LE-LMNN is applied on CohnKanade database [35], one of the most comprehensive

Table 1. Confusion matrix of seven-class facial emotions recognition using LE-LMNN proposed approach (JAFFE Database).

\begin{tabular}{|c|c|c|c|c|c|c|c|}
\hline & Anger $(\%)$ & Disgust $(\%)$ & Fear $(\%)$ & Joy (\%) & Sadness $(\%)$ & Surprise $(\%)$ & Neutral (\%) \\
\hline Anger & 92.6 & 2.4 & 0 & 0 & 2.5 & 0 & 2.5 \\
\hline Disgust & 0 & 94.4 & 0.8 & 0 & 0 & 0 & 4.8 \\
\hline Fear & 0 & 0 & 91.0 & 6.0 & 0.5 & 0 & 2.5 \\
\hline Joy & 0 & 0 & 1.4 & 96.0 & 0 & 0 & 2.6 \\
\hline Sadness & 2.1 & 0 & 0 & 0 & 94.2 & 1.0 & 2.7 \\
\hline Surprise & 0 & 0 & 0 & 0.2 & 0 & 96.5 & 3.3 \\
\hline Neutral & 0 & 2.3 & 0 & 0 & 1.5 & 0 & 96.2 \\
\hline
\end{tabular}

Bold values indicate the highest value of observation among the different methods 
Table 2. Comparison of recognition performance of reported methods and LE-LMNN proposed approach (JAFFE database).

\begin{tabular}{lc}
\hline Method (feature + classifier) & Seven-class recognition (\%) \\
\hline Advanced LBP + NLDA [37] & 85.6 \\
LBP + LP [36] & 93.8 \\
LE-LMNN (proposed approach) & $\mathbf{9 4 . 5}$ \\
\hline
\end{tabular}

Bold value indicates the highest value of observation among the different methods

databases in the current research area. Here, 320 image sequences are selected and cropped into smaller size of $100 \times 100$ pixels. The selection of the sequence is based on the seven emotions. These sequences come from 96 subjects, with seven-emotions per subject. For fair comparison with existing techniques [15] 1280 images (108 Anger, 120 Disgust, 99 Fear, 282 Joy, 126 Sadness, 225 Surprise, and 320 Neutral) are considered for the test. In literature, most of the performances are based on the 10 -fold cross-validation testing scheme. Therefore, a 10 -fold cross-validation scheme is adapted in this experiment. In this, the entire dataset is partitioned into 10 groups of approximately equal number of images and nine groups are used as a training database to train the neural network classifier whereas one group is used as a test dataset. The LM-based learning neural network is used to classify the emotions having the same configuration as given in section 4.1. Table 3 shows the confusion matrix of seven-class facial expression recognition. In this experiment, disgust, joy, surprise and neutral are recognized with high accuracy (98.7-99.7\%) while the recognition rates for anger, fear and sadness are comparatively lower (93.1-94.5\%).

Recently, Shan et al [15] conducted the various experimentations using different LBP representation with SVMs (RBF) on Cohn-Kanade database. Boosted-LBP performed best among the LBP based method, achieving 91.4\% recognition rate whereas the LE-LMNN proposed method achieves highest recognition rate of $96.47 \%$. Thus, the proposed method performs better than the methods reported in literature. The comparison of various reported methods and LE-LMNN proposed method is summarized in table 4.
It is also observed that the recognition rate for CohnKanade database is higher than the JAFFE database. This is due to the less number of images in the JAFFE database. Thus, the LM-based neural network performed better and models the input-output relationship to the desired accuracy for larger datasets.

The LE-LMNN proposed method is implemented using MATLAB R2011a on an Intel(R) Core(TM) i3 CPU with 2.4 GHZ frequency and 4 GB RAM. The memory space required for feature dimensions as well as computational time required for feature extraction process by LBP operator, Gabor filter and proposed approach is also compared. The eigenvalues in the proposed approach are calculated using the covariance matrix as well as Singular Value Decomposition (SVD) which contribute most of the computational complexity in the calculation. The computation of the covariance matrix [A] of a $N \times N$ pixels image requires multiplications and summation of the order $O(N)^{3}$. Moreover, for SVD operation, the computational complexity is also of the order $O(N)^{3}$ [4]. However, the computational complexity reduced dramatically in classification phase as proposed algorithm needs only six eigenvalues. The comparison of various existing methods and proposed approach is shown in table 5. It is observed from table 5 that the proposed approach has the significant speed and requires very less storage memory space as compared to the reported methods. Thus, the LE-LMNN method proves to be superior in terms of memory space and speed.

Table 4. Comparison of registration performance of reported methods and LE-LMNN proposed approach (Cohn-Kanade database).

\begin{tabular}{lc}
\hline Method (feature + classifier) & Seven-class recognition (\%) \\
\hline Geometric feature + TAN [22] & 73.2 \\
LBP + template matching [15] & $79.1 \pm 4.6$ \\
Gabor + SVM [15] & $86.9 \pm 3.5$ \\
LBP + SVM [15] & $88.9 \pm 3.5$ \\
Boosted-LBP + SVM [15] & $91.4 \pm 3.8$ \\
LE-LMNN (proposed approach) & $\mathbf{9 6 . 4 7}$ \\
\hline
\end{tabular}

Bold value indicates the highest value of observation among the different methods

Table 3. Confusion matrix of seven-class facial expression recognition using LE-LMNN proposed approach (Cohn-Kanade Database).

\begin{tabular}{lccccccc}
\hline & Anger $(\%)$ & Disgust $(\%)$ & Fear $(\%)$ & Joy $(\%)$ & Sadness $(\%)$ & Surprise $(\%)$ & Neutral $(\%)$ \\
\hline Anger & $\mathbf{9 4 . 0}$ & 0.9 & 0 & 0 & 2.5 & 0 & 2.6 \\
Disgust & 0 & $\mathbf{9 8 . 7}$ & 0 & 1.1 & 0 & 0 & 0.2 \\
Fear & 0 & 0 & $\mathbf{9 3 . 1}$ & 4.2 & 1.5 & 0 & 0 \\
Joy & 0 & 0 & 0 & $\mathbf{9 9 . 6}$ & 0 & 0 & 0.4 \\
Sadness & 3.2 & 0 & 0 & 0 & 0 & $\mathbf{9 4 . 5}$ & 0 \\
Surprise & 0 & 0 & 0 & 1.3 & 0 & $\mathbf{9 9 . 7}$ & 0 \\
Neutral & 0 & 0 & & 0.3 & 0 & $\mathbf{9 8 . 7}$ \\
\hline
\end{tabular}

Bold value indicates the highest value of observation among the different methods 
Table 5. Memory space and computational time for extracting LBP, Gabor-filter and Eigenvalue based features.

\begin{tabular}{lccc}
\hline & Gabor feature [15] & LBP feature [15] & LE-LMNN (proposed approach) \\
\hline Memory (Feature dimension) & 42,650 & 2478 & 6 \\
Time (feature extraction) (second) & 30 & 0.030 & 0.018 \\
\hline
\end{tabular}

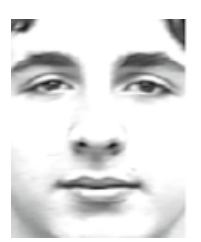

$110 \times 150$

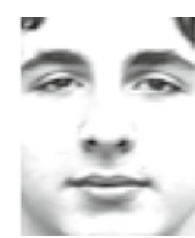

$55 \times 75$

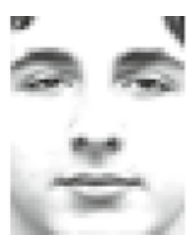

$36 \times 49$

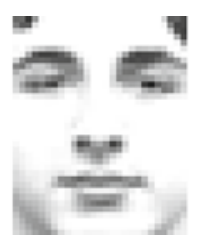

$27 \times 37$

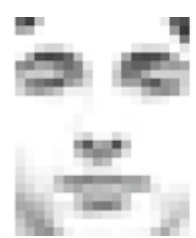

$18 \times 25$

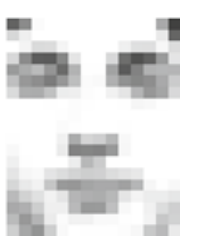

$14 \times 19$

Figure 8. The sample low resolution face expression images from Cohn-Kanade database.

Table 6. Recognition performance (\%) in varying resolution of LE-LMNN approach and reported methods.

\begin{tabular}{|c|c|c|c|c|c|c|}
\hline Pixels size & $110 \times 150$ & $55 \times 75$ & $36 \times 49$ & $27 \times 37$ & $18 \times 25$ & $14 \times 19$ \\
\hline LE-LMNN & 97.4 & 96.5 & 95.4 & 94.2 & 93.22 & 91.5 \\
\hline LBP & 92.6 & 88.9 & 87.3 & 84.3 & 79.6 & 76.9 \\
\hline Gabor & 92.2 & 91.6 & - & 77.6 & - & 68.2 \\
\hline Feature tracking & 91.8 & 91.6 & - & - & - & - \\
\hline
\end{tabular}

\subsection{Evaluation of varying resolution}

Tian [20] and Shan et al [15] conducted the various experimentations on six different face resolutions $(110 \times 150,55 \times 75,36 \times 48,27 \times 37,18 \times 24$ and $14 \times 19$ pixels). These images are down sampled and cropped from the original Cohn-Kanade database. The value of $\Lambda_{n}$ increases slightly as the resolution of an image decreases and therefore, the recognition performance is less affected by the change in resolution (91.5-97.4\%). The same image database as considered by Tian [20] and Shan et al [15] is used in this experiment and shown in figure 8 .

The experimentations are carried out using the LELMNN approach and recognition performance is compared with different reported methods as shown in table 6 . It is observed from table 6 that the recognition performance of LE-LMNN is less affected by the reduction in resolution, whereas the recognition performance of the methods available in literature reduces drastically for lower resolutions. Hence, the LE-LMNN approach for facial expression recognition proves to be an efficient and robust alternative to the methods available in literature.

\section{Conclusion}

In this paper, a new facial emotion recognition method based on the larger eigenvalues and Levenberg-Marquardt-based classifier (LE-LMNN) is proposed. The facial features are represented by few eigenvalues of diagonal matrix and therefore, reduce the memory space and the computational complexity. This technique is invariant to contrast as the test images are normalized. The proposed approach is examined on most popular and publicly available JAFFE and Cohn-Kanade databases. The various experimentations show that LE-LMNN proposed approach is more effective and computationally efficient for all seven-facial expressions recognition. It is concluded that the recognition performance of the proposed approach is significantly higher than the existing facial expression recognition methods. Moreover, the LELMNN method is more robust over the considerable range of low resolution of face images.

\section{References}

[1] Mehrabian A 1971 Silent messages. Wadsworth Publishing Company, Inc. Belmont, California

[2] Moore S and Bowden R 2011 Local binary patterns for multi-view facial expression recognition. Comput. Vis. Image Underst. 115: 541-558

[3] Tian Y, Kanade T and Cohn J 2005 Facial expression analysis. Handbook of face recognition, Springer (chapter 11)

[4] Gaidhane V H, Hote Y V and Singh V 2014 An efficient approach for face recognition based on common eigenvalues. Pattern Recognit. 47: 1869-1879

[5] Fasel B and Luttin J 2003 Automatic facial expression analysis: A survey. Pattern Recognit. 36: 259-275 
[6] Sánchez A, Ruiz J V, Moreno A B, Montemayor A S, Hernández J and Pantrigo J J 2011 Differential optical flow applied to automatic facial expression recognition. Neurocomputing 74: 1272-1282

[7] Yacoob Y and Davis L S 1996 Recognizing human facial expression from long image sequences using optical flow. IEEE Trans. Pattern Anal. Mach. Intell. 18: 636-642

[8] Pantic M and Rothkrantz L 2000 Expert system for automatic analysis of facial expression. Image Vis. Comput. 18: 881-905

[9] Tian Y L, Kanade T and Cohn J 2001 Recognizing action units for facial expression analysis. IEEE Trans. Pattern Anal. Mach. Intell. 23: 1-19

[10] Yang, J, Zhang D, Frangi A F and Yang, J Y 2004 Twodimensional PCA: A new approach to appearance-based face representation and recognition. IEEE Trans. Pattern Anal. Mach. Intell. 26: 131-136

[11] Eftekhari A, Forouzanfar M, Moghaddam H A and Alirezaie J 2010 Block-wise 2D Kernel PCA/LDA for face recognition. Inf. Process. Lett. 110: 761-767

[12] Li M and Yuan B 2005 2D-LDA: A statistical linear discriminant analysis for image matrix. Pattern Recognit. Lett. 26: $527-532$

[13] Ekenel H K and Sankur B 2004 Feature selection in the independent component subspace for face recognition. Pattern Recognit. Lett. 25: 377-1388

[14] Bashyal S and Venayagamoorthy G K 2008 Recognition of facial expressions using gabor wavelets and learning vector quantization. Eng. Appl. Artif. Intell. 21: 1056-1064

[15] Shan C, Gong S and Mcowan P W 2009 Facial expression recognition based on local binary patterns: A comprehensive study. Image Vis. Comput. 27: 803-816

[16] Ojala T, Pietikäinen M and Harwood D 1996 A comparative study of texture measures with classification based on feature distribution. Pattern Recognit. 29: 51-59

[17] Ojala T, Pietikäinen M and Mäenpää T 2002 Multiresolution gray-scale and rotation invariant texture classification with local binary patterns. IEEE Trans. Pattern Anal. Mach. Intell. 24: 971-987

[18] Pietikäinen M, Hadid A, Zhao G and Ahonen T 2011 Computer vision using local binary patterns, vol. 40, Springer

[19] Padgett C and Cottrell G 1997 Representing face images for emotion classification. Adv. Neural Inf. Proc. Syst. 9: 894-900

[20] Tian Y 2004 Evaluation of face resolution for expression analysis. In: Proc. CVPRW'04, pp. 82-82

[21] Lee B, Chun J and Park P 2008 Classification of facial expression using SVM for emotion care service system. In: Proceedings of SNPD'08, pp. 8-12
[22] Cohen I, Sebe N, Garg A, Chen L and Huang T S 2003 Facial expression recognition from video sequences: Temporal and static modelling. Comput. Vis. Image Underst. 91: 160-187

[23] Pantic M and Rothkrantz L 2004 Facial action recognition for facial expression analysis from static face images. IEEE Trans. Syst. Man Cybern. Part B Cybern. 34: 1449-1461

[24] Pantic M and Rothkrantz L M 2000 Automatic analysis of facial expressions: The state of the art. IEEE Trans. Pattern Anal. Mach. Intell. 22: 1424-1445

[25] Pantic M and Patras I 2006 Dynamics of facial expression: recognition of facial actions and their temporal segments from face profile image sequences. IEEE Trans. Syst. Man Cybern. Part B Cybern. 36: 433-449

[26] Wee C Y and Parmesran R 2007 Measure of image sharpness using eigenvalues. Inf. Sci. 177: 2533-2552

[27] Bie T D, Cristianini N and Rosipal R 2005 Eigenproblems in pattern recognition. Handbook of Geometric Computing, Springer Berlin Heidelberg, pp. 129-167

[28] Agarwal M, Jain N, Kumar M and Agrawal H 2010 Face recognition using eigen faces and artificial neural network. Int. J. Comput. Theor. Eng. 2: 1793-8201

[29] Gaidhane V H, Hote Y V and Singh V 2012 Nonrigid image registration using efficient similarity measure and Levenberg-Marquardt optimization. Biomed. Eng. Lett. 2: 118-123

[30] Gaidhane V H, Hote Y V, Singh V and Kumar M 2011 New approaches for image compression using neural network. $J$. Intell. Learning Syst. Appl. 3: 220-229

[31] Gaidhane V, Singh V and Kumar M 2010 Image compression using PCA and improved technique with MLP neural network. In: Proceedings of ARTCOM'10, Kottayam, India, pp. $106-110$

[32] Singh V, Gupta I and Gupta H O 2005 ANN-based estimator for distillation using Levenberg-Marquardt approach. Eng. Appl. Artif. Intell. 20: 249-259

[33] Hagan M T, Demuth H B and Beale M 2003 Neural network design. 7th International Student Edition. Vikas Publishing House

[34] Lyons M J, Budynek J and Akamatsu S 1999 Automatic classification of single facial images. IEEE Trans. Pattern Anal. Mach. Intell. 21: 1357-1362

[35] Kanade T, Cohn J F and Tian Y 2000 Comprehensive database for facial expression analysis. In: Proceedings of AFGR'00, Grenoble, France, pp. 46-53

[36] Feng X, Pietikäinen M and Hadid T 2005 Facial expression recognition with local binary patterns and linear programming. Pattern Recognit. Image Anal. 15: 546-548

[37] Liao S, Fan W, Chung A C S and Yeung D Y 2006 Facial expression recognition using advanced local binary patterns, Tsallis entropies and global appearance features. In: Proceedings of ICIP'06, Atlanta, GA, pp 665-668 